\title{
Implication of a $P-L$ Relation of Mira Variables
}

\author{
Hiromoto Shibahashi \\ Department of Astronomy, University of Tokyo, Japan
}

Feast et al. (1989) obtained observationally a period-luminosity ( $P-L)$ relation of Mira variables in LMC. Basically, fundamental periods of stellar pulsation should be determined by two quantities - a mass and a radius of the star. The existence of a $P-L$ relation implies a special condition that reduces a function of two variables into a function of only a single variable. Though the AGB appears as a thin line in the HR diagram like the Cepheid instability strip, it is an "asymptotically" merged line of evolutionary tracks of stars in a range of about $0.5-3$ solar masses. This fact means that the masses of stars on a point of the AGB are not unique though the radii and the effective temperatures are unique, respectively. Therefore we cannot expect, in the case of AGB stars, that a period is reduced to a function of a single variable. The periods of an AGB star should be a function of two variables - the luminosity and the mass of the star. The observationally obtained $P-L$ relation should be interpreted as such a period-luminosity-mass relation, and scattering of the relation must be recognized as reflection of the mass range of Mira variables.

The luminosity of an AGB star is strongly dependent on the core mass and almost independent of the total mass of the star. However, the pulsation period is dependent on the total mass of a star, and becomes shorter with increasing mass for a given luminosity (and hence a given effective temperature). This fact means that we would be able to distinguish masses of AGB stars from evolutionary tracks drawn on a $(P, L)$-diagram while we cannot do so from evolutionary tracks in the HR diagram. Preliminary calculations show that an evolutionary track of a star on a $(P, L)$-diagram is less steeply inclined than the observationally fitted period-luminosity relation of Mira variables. If we plot the observed period and luminosity of each Mira variable and draw evolutionary tracks of various mass stars on the same $(P, L)$-diagram, we can determine the mass of each star. If we can deduce the mass and the radius of each Mira variable in this way, we can determine the position of the star in the HR diagram by mapping again a theoretical (mass, radius)-diagram onto the HR diagram. The distribution of Mira variables on a $(P, L)$-diagram has a little bit steeper inclination than an evolutionary track of a star. This feature is conserved by mapping the $(P$, $L)$-diagram onto the HR diagram. Hence, the distribution of Mira variables on the HR diagram must be inclined with respect to the asymptotic giant branch from the left above to the right below. This means that Mira variables concentrate in a more specific region of the HR diagram, and implies the existence of an instability region for Mira variables.

\section{References:}

Feast, M.W., Glass, I.S., Whitelock, P.A., and Catchpole, R.M. 1989, MNRAS, 241, 375. 